\title{
La fecundidad bajo el reemplazo y las políticas familiares en América Latina y el Caribe: qué puede aprenderse de la experiencia europea
}

\author{
Ignacio Pardo \\ Carmen Varela*
}

El descenso de la fecundidad en América Latina y el Caribe es un fenómeno inédito y extremadamente veloz. Hoy, numerosos países de la región tienen fecundidad bajo el nivel de reemplazo, incluyendo casos emblemáticos como el de Brasil. Aquí se debate desde qué marco conceptual se pueden comprender las diferentes medidas, programas o políticas que están surgiendo y surgirán en el futuro en relación a temas de fecundidad y familia. Además, se resumen e interpretan las experiencias de aquellos países en los que se han implementado políticas familiares en contexto de fecundidad bajo el reemplazo (concretamente, las políticas implementadas en Suecia, España y Francia) discutiendo de qué manera las experiencias europeas pueden resultar útiles para la formulación de políticas en América Latina. Para concluir, se enumeran las decisiones clave a tomar para el diseño de políticas familiares en contexto de baja fecundidad.

Palabras clave: Fecundidad. Políticas familiares. Fecundidad bajo el reemplazo. América Latina y el Caribe.

\footnotetext{
* Programa de Población, Facultad de Ciencias Sociales, Universidad de la República, Montevideo, Uruguay (ipardo@fcs. edu.uy).

** Programa de Población, Facultad de Ciencias Sociales, Universidad de la República, Montevideo, Uruguay (cvarela@ fcs.edu.uy).
} 


\section{Introducción}

En las últimas décadas, los países latinoamericanos han experimentado un descenso sostenido de su fecundidad. Significativamente, la Tasa Global de Fecundidad está situada en varios países por debajo del nivel de reemplazo poblacional (Tabla 1), incluyendo casos emblemáticos como Brasil, aunque esta medición agregada contenga heterogeneidades al interior de los países, con algunas subpoblaciones que aún presentan niveles altos de fecundidad.

El escenario, por cierto, es inédito para la mayoría de la región (salvo casos aislados de transición demográfica extremadamente avanzada, como Barbados y Cuba, cuyas Tasas Globales de Fecundidad se encontraban bajo el reemplazo en los ‘80). De algún modo, todo lo que sabíamos acerca de la fecundidad en América Latina está siendo sometido a cambios y desafíos de gran importancia, tanto en lo académico como en lo social y político.

El descenso de la fecundidad en nuestra región, como en otras, se vincula con el cambio en las preferencias reproductivas (CASTERLINE \& MENDOZA, 2009), tal como se lo ha medido tradicionalmente, en términos de número ideal de hijos que declaran las mujeres, o aun con las mediciones más precisas, que distinguen "ideales" de "preferencias" en el comportamiento reproductivo. Aunque es multicausal, este proceso se vincula con la diversificación del proyecto de vida de las mujeres y su creciente incorporación al mercado laboral.

De hecho, los estudios más recientes ligan la preferencia por un tamaño menor de la descendencia con la existencia de cierta conflictividad entre el trabajo y la maternidad, provocado por la persistencia de roles de género que continúan asignando la carga principal de la crianza a las mujeres. En ese sentido, se ha observado correlación entre capacidad de control de los tiempos del propio trabajo y cantidad deseada de hijos (BEGALL; MILLS, 2011) y se han explorado relaciones más complejas, tales como la que existe entre cantidad deseada de hijos y percepción de los hombres acerca del conflicto entre trabajo y maternidad al que pueden estar sometidas sus parejas (SHREFFLER; PIRRETTI; DRAGO, 2010). En todos los casos, la hipótesis del peso de la difícil conciliación crianza - trabajo se vio reforzada.

Hasta hace poco, algunos autores suponían que los países de fecundidad más baja difícilmente revertirían su situación (REHER 2007). En algunos casos, el argumento partía de la hipótesis de la "low fertility trap", una suerte de círculo vicioso que integra factores económicos, de ideas y de estructura de la pirámide poblacional (las cohortes con baja TGF generan cohortes más pequeñas) que operaría en países con la TGF bajo el umbral de 1,5 hijos por mujer (LUTZ; SKIRBEKK; TESTA, 2006). ${ }^{1}$

\footnotetext{
${ }^{1}$ Sin embargo, la fecundidad se viene recuperando en algunos países que habían caído por debajo de una TGF de 1,5 hijos y presentaban así una fecundidad “lowest-low”. España, por ejemplo, tenía una TGF de 1,16 en 1998 y aumentó sus niveles hasta 1,46 en 2008; además, sucedió en muchas regiones de fecundidad extremadamente baja, como el País Vasco, donde era de 0,91 en 1994 y llegó a 1,33 en 2008, por lo que la hipótesis de la low fertility trap tiene menos sustento hoy que algunos años atrás. Esta recuperación de la TGF se debe sobre todo a un efecto de medición. Como la caída estaba dada en gran medida por el retraso en el calendario de la fecundidad y no exclusivamente por un descenso de su intensidad, los indicadores de momento (como la TGF), incapaces de discriminar calendario e intensidad, reflejaron una mayor caída en la fecundidad que los de cohorte.
} 
TABLA 1

Tasa global de fecundidad

Países de América Latina y el Caribe - 1980-2015

\begin{tabular}{|c|c|c|c|c|c|}
\hline País o región & $1980-1985$ & $\begin{array}{l}\text { Estimación } \\
2010-2015\end{array}$ & País o región & $1980-1985$ & $\begin{array}{r}\text { Estimación } \\
2010-2015 \\
\end{array}$ \\
\hline Antillas Neerlandesas & 2,4 & 1,9 & $\begin{array}{l}\text { Islas Vírgenes de los } \\
\text { Estados Unidos }\end{array}$ & 3,7 & 1,9 \\
\hline Argentina & 3,2 & 2,2 & Jamaica & 3,6 & 2,3 \\
\hline Aruba & 2,4 & 1,7 & México & 4,3 & 2,0 \\
\hline Bahamas & 3,1 & 1,9 & Nicaragua & 5,9 & 2,6 \\
\hline Barbados & 1,9 & 1,6 & Panamá & 3,5 & 2,4 \\
\hline Belice & 5,4 & 2,7 & Paraguay & 5,2 & 2,8 \\
\hline $\begin{array}{l}\text { Bolivia (Estado } \\
\text { Plurinacional de) }\end{array}$ & 5,3 & 3,1 & Perú & 4,7 & 2,4 \\
\hline Brasil & 3,8 & 1,7 & Puerto Rico & 2,5 & 1,8 \\
\hline Chile & 2,7 & 1,9 & República Dominicana & 4,2 & 2,5 \\
\hline Colombia & 3,7 & 2,3 & $\begin{array}{l}\text { San Vicente y las } \\
\text { Granadinas }\end{array}$ & 3,6 & 2,0 \\
\hline Costa Rica & 3,5 & 2,0 & Santa Lucía & 4,2 & 1,9 \\
\hline Cuba & 1,9 & 1,5 & Surinam & 3,7 & 2,3 \\
\hline Ecuador & 4,7 & 2,4 & Trinidad y Tobago & 3,2 & 1,6 \\
\hline El Salvador & 4,8 & 2,2 & Uruguay & 2,6 & 2,0 \\
\hline Granada & 4,2 & 2,2 & $\begin{array}{l}\text { Venezuela (República } \\
\text { Bolivariana de) }\end{array}$ & 4,0 & 2,4 \\
\hline Guatemala & 6,1 & 3,7 & & & \\
\hline Guyana & 3,3 & 2,2 & $\begin{array}{l}\text { América Latina y el } \\
\text { Caribe }\end{array}$ & 3,9 & 2,2 \\
\hline Haití & 6,2 & 3,2 & América Latina & 4,0 & 2,1 \\
\hline Honduras & 6,0 & 3,0 & El Caribe & 3,4 & 2,3 \\
\hline
\end{tabular}

Fuente: Cepal (2011) Anuario Estadístico de América Latina y el Caribe 2011.

Por cierto, la TGF de ningún país latinoamericano cayó por debajo de 1,5, pero el descenso de la fecundidad abre la puerta a tales debates y preguntas. La experiencia de los países de baja fecundidad (como la mayoría de los europeos) probablemente no se repetirá en los mismos términos en América Latina, pero de ella pueden extraerse hipótesis relevantes, como la postergación de la edad al primer hijo, el aumento de mujeres sin hijos y, en términos más generales, el tránsito hacia cursos de vida en los que la sucesión típica de eventos vuelve improbable el retorno a niveles ampliamente superiores al reemplazo (FREJKA; SARDON, 2006, 2004). De todos modos, existe una importante variedad entre los propios países europeos, en gran medida debido a sus distintos arreglos institucionales (ADSERA, 2011). También entre los países de nuestra región, por más que puedan tender a la convergencia en varias dimensiones.

El descenso de la fecundidad tiene dos connotaciones importantes. Por un lado, existe una creciente acumulación de investigación demográfica latinoamericana, que permite conjeturar acerca de las posibles características del cambio en el comportamiento reproductivo de la región (ROSERO-BIXBY et al., 2009). Por otro, las matrices de protección social de los estados 
latinoamericanos se han modificado, incorporando crecientemente medidas de política familiar que atienden estos temas. Entre otras medidas, aquellas tendientes a disminuir las diferencias en las relaciones de género y que promueven la redistribución de roles permitiendo al varón y la mujer combinar trabajo con crianza. En cualquier caso, los países latinoamericanos se encuentran en diferentes estadios de un mismo proceso de reelaboración de políticas. Las medidas ya no tendrán como foco único el exceso de fecundidad respecto de la deseada sino también el déficit o fecundidad deseada no materializada. Y las orientaciones de política ya no se limitarán solamente a la ampliación del acceso y uso eficiente de la anticoncepción, porque deberán incluir medidas más diversas y complejas destinadas a facilitar e incentivar la tenencia de hijos, en particular del primer o segundo hijo, entre quienes así lo desean, y, de paso, procurar evitar el advenimiento de un régimen de fecundidad muy baja.

La pregunta, por tanto, girará hacia un nuevo eje: ¿qué hacer, si es que hubiera que hacer algo, con el nuevo escenario de fecundidad bajo el reemplazo? En el nuevo contexto conviven elementos disímiles, como la reformulación de viejos discursos pronatalistas ${ }^{2}$ (que ya comienzan a ser fuertes en multitud de ámbitos, incluidos los gubernamentales, de varios de nuestros países), la vigencia del marco de Derechos Sexuales y Reproductivos de El Cairo y la consideración del potente cambio familiar experimentado en las últimas décadas.

Nuestro artículo parte de este escenario para desarrollar dos objetivos básicos. Por un lado, explicitar cuáles son las motivaciones, marcos conceptuales y las opciones concretas de programas, políticas y medidas que pueden implementarse, tomando en cuenta aquellas que llevaron adelante algunos países europeos, ${ }^{3}$ de forma de discutir su posible aplicabilidad en el contexto específico de América Latina. Por otro, enumerar las principales decisiones que deben tomarse ante la necesaria reformulación de las políticas vinculadas a la familia y la fecundidad.

\section{Antinatalismo, pronatalismo y más: qué motivación tienen las políticas}

En América Latina, como en otras regiones, la discusión suele trascender el equívoco concepto de políticas de fecundidad para situarse en el marco distinto y más amplio de las políticas familiares: son todas las medidas que doten de recursos a personas con responsabilidades familiares (haya vínculos de consanguinidad o no) para ayudarlas a desempeñar tareas vinculadas a esta condición, entre las que se destaca la de atender a los menores dependientes.

"En inglés se habla de "parenting policies" o políticas "family-friendly"... (aunque este último término) apela más bien a la necesidad de armonizar disposiciones ya existentes en lugar de diseñar nuevos tipos de intervenciones" (FLAQUER, 2000, p. 11). Los antecedentes

\footnotetext{
${ }^{2}$ Ya no se trata de los discursos contrarios a la planificación familiar ante el posible descenso de la fecundidad, sino de propuestas basadas en incentivos para revertir ese descenso

${ }^{3}$ La acumulación de experiencias en este tipo de políticas hace imprescindible considerar las iniciativas europeas como la principal referencia, pero futuros trabajos acerca de las políticas en América Latina podrían beneficiarse de la experiencia de otros países. Por ejemplo, de las políticas relacionadas a la fecundidad en Irán, donde el veloz descenso de la fecundidad obligó a revisar las medidas vinculadas al family planning y revertir la orientación de las políticas.
} 
datan de la década de 1930 (Alva Myrdal), aunque ya se habían aplicado algunas medidas de política familiar, como licencias de maternidad, en 1870 (GAUTHIER, 1996). Desde 1960, las políticas familiares cobraron especial interés, desde la preocupación por el descenso de la fecundidad de los países desarrollados.

Tradicionalmente, la caída de la fecundidad ha generado reacciones pronatalistas, vinculadas al nacionalismo y al eugenismo (ante la amenaza de menos o "peor" población para el país). Así, las políticas que intentaron aumentar la fecundidad partieron en más de una oportunidad de concepciones totalitarias, como en el caso del pronatalismo mussoliniano y la Alemania Nazi (ROSSY, 2011).

El origen de las políticas pronatalistas puede ubicarse en el siglo XIX francés, gracias a la constante comparación con el mayor tamaño de población de su vecino, Alemania. Así surgió a fines del siglo XIX un fuerte movimiento pronatalista, que acuñó el término dénatalité desde la preocupación por la tendencia al descenso de la fecundidad en Francia en el marco del revés militar frente a Alemania en la guerra de 1870. Hasta la década de 1960, al menos, estas políticas reforzaron el modelo del varón sustentador y la madre dedicada a los cuidados familiares: la asignación familiar se solía pagar desde el nacimiento del primer hijo, siempre que la mujer no trabajara. En las décadas más recientes, la mayor participación femenina en el mercado laboral fue volcando la balanza hacia el concepto de conciliación familiar - laboral.

Desde la concepción opuesta, el neomalthusianismo desde el que se intentó controlar el crecimiento poblacional se tradujo en un antipoblacionismo frecuentemente coercitivo (CONNELLY, 2008; ROBINSON; ROSS, 2007). Sobre todo entre los años 1950 y 1970, cuando se configuró un establishment de control poblacional, que con el tiempo generaría lo que Connelly (2008) Ilama un "sistema sin cerebro": una red de filántropos, investigadores, asociaciones internacionales, agencias gubernamentales y voluntarios en la que las buenas intenciones y las esterilizaciones forzosas convivían bajo un mismo impulso de escala global.

En la década de 1960, la red de instituciones que lideraron este movimiento tuvo su período de mayor fortaleza. La mirada más oscura desde esta perspectiva es aquella en la que se percibe una perspectiva eugenista que no se presenta explícitamente como tal sino que surge como caballo de Troya dentro de perspectivas más "aceptables". ${ }^{4}$ Esta impronta del antinatalismo ya no es mayoritaria, aunque aún se la puede rastrear en el discurso público acerca de las políticas de fecundidad o familiares. ${ }^{5}$ En las décadas de 1970 y 1980 , los gobiernos comenzaron a contrarrestar los argumentos y prácticas del movimiento de control poblacional. Por cierto, India y China generaron sus políticas al respecto (en el

\footnotetext{
${ }^{4}$ Para este "eugenismo encubierto" se ha propuesto el término inglés “crypto - eugenics" (CONNELLY, 2008)

${ }^{5}$ De hecho, la idea de "bomba poblacional” (EHRLICH, 1968) no tuvo éxito porque describía adecuadamente las tendencias poblacionales, sino porque fue una calificada intervención sobre la opinión pública. En su libro, Ehrlich logró impactar al público al partir de una experiencia personal: su viaje a la India en 1966, donde convivió con grandes multitudes. Por cierto, como bien apunta Connelly (2008), Ehrlich podría haber encontrado mayores multitudes en la Nueva York o Londres de esos años; el impacto que el autor quiso transmitir fue más bien por la "calidad": la raza y la pobreza de la población, que se diseminarían por el mundo en caso de no controlar la cantidad de hijos que tienen "cierto tipo" de mujeres.
} 
caso de China más draconianas que las preconizadas por el movimiento global de control poblacional), pero el control de los nacimientos ya no sería de recibo como objetivo de "la humanidad”. Finalmente, la Conferencia de El Cairo en 1994 fue el golpe de gracia para los intentos controlistas. A partir de allí, la perspectiva de derechos fue el paradigma prevalente, aunque las políticas de fecundidad siguiesen abrevando hasta hoy en diferentes miradas, no siempre ajustadas a la perspectiva de derechos de forma plena.

De todos modos y más allá de orígenes, las políticas familiares pueden tener objetivos pronatalistas tanto como neomalthusianos, defensores de la familia tradicional y patriarcal tanto como favorables a los arreglos familiares más diversos y equitativos. En definitiva, el modelo de familia y de sociedad que se promueve no es ajeno a las medidas que se tomen: en el contexto actual, el desarrollo de políticas familiares en el contexto del respeto a los Derechos Humanos y el fomento de la autonomía y el bienestar de las personas no sólo es posible sino que es deseable y cuenta casi con consenso global (aunque no unanimidad) en su favor.

Existen críticas, aunque algunas son fácilmente rebatibles o parten de premisas problemáticas. Se argumenta, por ejemplo, que si se fomenta la fecundidad en general, tendrán más hijos las personas “equivocadas” (pobres, adolescentes) (MCDONALD, 2006, p. 217). Lo cierto es que si se procede como en Singapur, donde se concentraron los incentivos en ciertos sectores, más ricos o educados, el pronatalismo adopta un sesgo regresivo o racista. También se argumenta que una política así es una intromisión en la esfera privada de las personas, que la transferencia de recursos desde los que no tienen hijos hacia quienes sí los tienen es ilegítima, o que cualquier esfuerzo pronatalista es inútil y, por tanto, un derroche de recursos.

En cualquier caso, en los países latinoamericanos suele predominar una idea compartible: la de una necesaria y legítima intervención estatal en materia de políticas familiares, desde la perspectiva de derechos. A menudo, con la idea explícita de modificar los patrones de fecundidad de la población. Pero los debates comienzan con las preguntas posteriores: si cabe tener políticas familiares, ¿cuál debiera ser su orientación, cuál su fin último y cómo debiera procederse para alcanzarlo? Para eso, nos parece útil recurrir a la experiencia reciente de las políticas familiares europeas.

\section{Los modelos de política en la experiencia europea}

Las lecciones a extraer de la peripecia de los países desarrollados con políticas familiares más estables son diversas. Si bien durante mucho tiempo se resumió su situación puntualizando que los países con mayor porcentaje de empleo femenino eran aquellos con mayor recuperación de su fecundidad (y menores niveles de pobreza), lo que se interpretaba como un logro de las políticas que esos mismos países llevaban adelante, las valoraciones acerca de las medidas concretas y los criterios de construcción de tipologías de modelos de política no son unívocos. 
Los estados difieren en su institucionalidad y en el énfasis dado a cada uno de los objetivos más frecuentes: lograr la autonomía de los adultos, la educación de los niños y la equidad de género (THÈVENON, 2011). Más allá de la utilidad de las clasificaciones habituales de modelos de política familiar y formas de "desfamiliarizar" la crianza (ESPING-ANDERSEN, 2004), se requiere una revaloración permanente de las experiencias existentes. ${ }^{6}$ En este caso, para tomar en cuenta la experiencia europea pensando en el contexto latinoamericano, es útil reparar en tres modelos que ejemplifican diversas opciones disponibles:

- el basado en la equidad de género, a partir del ejemplo de Suecia;

- el basado en los cuidados infantiles e inspirado en orientaciones pronatalistas, a partir del ejemplo de Francia;

- el que contempla medidas de diverso tipo, incluidas transferencias económicas, a partir del ejemplo de España hasta 2010.

Estos modelos pueden describirse en pocas palabras. En los casos de Suecia (con persistente voluntad de socializar el costo de la crianza de los niños) y Francia (con una gran institucionalización de la política familiar), la estructura estatal cuenta con un conjunto de instituciones para el diseño y aplicación de políticas, así como existen organizaciones de la sociedad civil que actúan como grupos de interés. La fecundidad de ambos países es de las más altas de Europa. En España, por el contrario, ejemplo de fecundidad muy baja, la política familiar se encuentra fragmentada entre distintas medidas dirigidas a objetivos o ámbitos diversos (mujer, infancia, juventud, vivienda, mercado de trabajo, sistema fiscal) (FLAQUER, 2000).

En el caso de Suecia, la orientación de las políticas ha estado dirigida desde el comienzo a la equidad de género (sin intentar llevar la TGF a ningún nivel establecido a priori ni fomentando la crianza familiar de los niños) y permitiendo al hombre y la mujer combinar trabajo con crianza, en un contexto de pleno empleo que asegura sustentabilidad financiera. Las tasas de ocupación femenina están entre las más altas de Europa.

Se ha argumentado que los resultados de la fecundidad sueca, relativamente alta en comparación con los demás países europeos, son en parte el reflejo de una política familiar activa y de una cultura política amigable a las mujeres (woman-friendly political culture) (OLÁH; BERNHARDT, 2008; BJÖRKLUND, 2006; HOEM, 2005). Aquí, las políticas familiares y de equidad de género no son entidades separadas, sino que están entrelazadas y construidas para soportarse mutuamente. Y el objetivo expreso de la política de equidad de género es permitir al hombre y a la mujer combinar trabajo con crianza, por lo que no existen políticas familiares que apunten a apoyar el cuidado de los hijos sin más, o mantener la tasa de fecundidad a determinado nivel a priori. Por otra parte, desde la educación sexual escolar, que

\footnotetext{
${ }^{6}$ Por cierto, la necesidad de intervenciones está en debate. En nuestra opinión, mayoritaria también entre la mayoría de los gobernantes actuales de la región, la baja fecundidad en los países de América Latina merece la aplicación de medidas que desfamiliaricen la crianza. Los motivos son la búsqueda de equidad en el confort de los niños, la equidad de género y la satisfacción con la fecundidad (dada la existencia de insatisfacción por exceso y por defecto). En menor medida, se han esgrimido argumentos vinculados a la necesidad de prevenir la llegada a niveles de fecundidad "lowest-low", que acaso aceleran el envejecimiento poblacional, posiblemente problemático en un contexto de importante desigualdad y escasez de recursos.
} 
no se concentra en el matrimonio, se enfatiza el valor de las relaciones personales duraderas e íntimas. Hay una actitud positiva hacia las relaciones sexuales entre los jóvenes, incluso entre los que no corresiden, y no se recomienda la abstención como un medio para evitar embarazos no deseados (OLÁH; BERNHARDT, 2008). La tasa de fecundidad adolescente es muy baja.

A pesar de la dificultad inherente a la evaluación de las políticas familiares, los expertos suelen vincular las políticas familiares con las recientes tendencias de la fecundidad sueca, alta para su contexto (HOEM, 2005). Las políticas habrían contribuido a la postergación de la edad al primer hijo, la reducción de los intervalos intergenésicos y el aumento de la TGF hasta los actuales valores, cercanos al nivel de reemplazo (1,90 en 2011). Entre otras cosas, es importante que para las mujeres suecas entre 20 y 34 años el ideal sea de 2,4 hijos por mujer; un valor que se encuentra entre los más altos de Europa (OLÁH; BERNHARDT, 2008).

Por otra parte, Francia, con una gran institucionalización de la política familiar, combina objetivos pronatalistas con otros de asistencia familiar, de tal modo que la sociedad francesa y el sistema político aceptan sin objeciones la intervención del Estado en la esfera familiar. A diferencia de Suecia, orienta fundamentalmente sus políticas hacia la conciliación entre la vida familiar y laboral de las mujeres. Se destaca fundamentalmente la batería de medidas de cuidados infantiles.

Es uno de los países europeos con mayor fecundidad. Desde 1975 su TGF se ha mantenido bastante estable (alrededor de 1,9 hijos por mujer) y desde 1996, su tendencia es creciente, a diferencia de la que muestran la mayoría de los países europeos. Al igual que Suecia, este desempeño se conjuga con la incorporación en la sociedad francesa de los valores y características propios de la Segunda Transición Demográfica ${ }^{7}$. Nuevamente, con todas las prevenciones del caso, distintos autores interpretan que la explicación más probable para la fecundidad relativamente alta de Francia es una exitosa política familiar (FLAQUER, 2000; GAUTHIER, 2007; THÉVENON, 2009).

Finalmente, puede extraerse del caso de España un modelo diferente. Se trata de un país con mayor impronta familista, donde los miembros del hogar (y mayoritariamente sus mujeres) asumen tradicionalmente el cuidado de los niños. No existen políticas familiares tan integrales como en los dos casos anteriores, sino políticas fragmentadas para los distintos actores sociales (FLAQUER, 2000). En esa dispersión se han experimentado marchas y contramarchas, con medidas dirigidas a distintos actores sociales (mujer, infancia, tercera edad).

Una de las medidas más claras en torno a la fecundidad involucró el apoyo financiero: el "cheque bebé” (una transferencia universal y única, efectuada al momento del nacimiento

\footnotetext{
${ }_{7}^{7}$ Segunda Transición Demográfica es el nombre dado a una serie de fenómenos demográficos, vinculados fundamentalmente a los patrones de nupcialidad y fecundidad (inicialmente restringidos a la experiencia de los países desarrollados desde la década del '70): incremento de la soltería; incremento de la edad media a la primera unión conyugal, postergación de la maternidad/paternidad, expansión de las uniones consensuales, los nacimientos fuera del matrimonio y los divorcios, diversificación de la estructura de hogares y caída del nivel de fecundidad inferior al nivel de reemplazo, entre otros. El término surge de Lesthaeghe, R. e Van de Kaa (1986).
} 
del niño). El 31 de diciembre de 2010 fue retirada, no sólo para disminuir los costos del Estado, sino porque su propia eficiencia se encontraba en duda. La población, con una baja tasa de participación laboral femenina, tiene una de las fecundidades más bajas de Europa. A pesar de las diversas acciones que han tomado los sucesivos gobiernos, la tendencia de la fecundidad no se ha revertido. En nuestra opinión, el posible fracaso del modelo de políticas no está dado por la escasa intensidad de la fecundidad sino por otro indicador, más significativo: es uno de los países con mayor nivel de insatisfacción entre los hijos tenidos e hijos deseados (BERNARDI, 2005).

En cualquier caso, acercamientos como éste son todo lo que podemos tener en términos de una evaluación de políticas en el caso europeo: sería deseable contar con evaluaciones de impacto que midiesen su efectividad a partir de condiciones más rigurosas, acaso con diseños experimentales o cuasiexperimentales, pero tal cosa no es posible. ¿Quiere decir que debemos implementar políticas sin tener la certeza absoluta de cuán efectivas han resultado en el contexto europeo? Sí, pues las características de estos programas (a menudo de cobertura universal, sin la posibilidad de grupos de control y con una alta incapacidad de aislar el efecto de unas variables en relación a otras) han hecho difícil la realización de evaluaciones de impacto como las que serían deseables.

De todos modos, las evaluaciones de resultados que se han llevado adelante mencionan efectos que no logran ser más que moderados y puntuales, si se trata de cambios en la intensidad de la fecundidad, al menos cuando se trata de programas que actúan de forma aislada. En todo caso, lo sugerido más arriba para el caso de Suecia puede mantenerse en términos generales: los mejores resultados (prevención de la caída hacia niveles "lowestlow”, mayor satisfacción con la fecundidad, mejores condiciones de crianza en términos de corresponsabilidad colectiva) se han dado allí donde existen abordajes multidimensionales y sostenidos en el tiempo, generando previsibilidad en las condiciones en las que ejerce la paternidad y maternidad, a través del efecto conjunto de provisión de servicios de cuidados infantiles y ampliación de tiempos y espacios para la crianza durante todas las etapas de la niñez (TAKAYAMA; WERDING, 2009; THÉVENON, 2009; GAUTHIER, 2007).

\section{La especificidad latinoamericana y el aprovechamiento de la experiencia europea}

Así las cosas, ¿vale la pena tomar en cuenta estas experiencias? Nuestra respuesta es que sí, pues estos criterios pueden guiar mínimamente las decisiones más importantes de política pública, aun cuando no se trate de evaluaciones de impacto en sentido estricto. ¿Cuán aplicables son al contexto latinoamericano? Allí comienza otra discusión, quizá la más importante. A pesar del distinto nivel de desarrollo, hay algunas características de nuestros países, sobre todo en cuanto a sus comportamientos y estructuras demográficas, que comienzan a hacerlos comparables con algunos de los países europeos mencionados. Sin ir más lejos, los propios niveles de fecundidad, el porcentaje de población viviendo en ciudades y la tasa de actividad femenina. 
Sin embargo, la estructura dual de los comportamientos demográficos, propia de los países de nuestra región, amerita la generación de políticas que no actúen sólo sobre los promedios poblacionales, más útiles para contextos de menor heterogeneidad en los comportamientos. En nuestros países, las mujeres de los contextos socioeconómicos más desfavorables tienen una menor probabilidad de estar económicamente activas y una mayor cantidad de hijos en promedio. Y en dichos sectores, la fecundidad no deseada "por exceso" es aún un problema importante (PERI; PARDO, 2008), más allá del éxito de los programas de familiy planning y salud sexual y reproductiva desarrollados en las últimas décadas. Este fenómeno de tan alta proporción de personas con fecundidad no deseada no representa una carga cuantitativamente importante en los patrones de fecundidad de los países desarrollados, por lo que es necesario incorporarlo a las políticas de nuestra región de forma específica.

Además, las propias características de familia y fecundidad que solemos resumir en los indicadores de la Segunda Transición Demográfica son idiosincrásicas. La cohabitación, por ejemplo, presenta características históricas que la hacen diferir en su significado respecto de la versión europea del fenómeno; el correlato empírico más visible de esta diferencia es su histórica prevalencia entre los sectores de menor nivel educativo, contrariamente a lo que sucede en los países desarrollados. Esto abre la puerta a distintas interpretaciones sobre su actual "boom" (ESTEVE et al., 2012) y a debates sobre la valoración del alto porcentaje de fecundidad no matrimonial (CASTRO MARTIN et al., 2011).

El descenso de la fecundidad en América Latina se ha dado, por otra parte, a una velocidad superior a la de los países pioneros de la transición demográfica, donde en pocos años pudo observarse un aumento sustantivo de las mujeres de 30 años que no son madres, sobre todo entre las más educadas (ROSERO-BIXBY et al., 2009). Los dos posibles mecanismos demográficos para esto (mayor prevalencia de las mujeres sin hijos en las cohortes actuales o aplazamiento del momento de tenerlos) se combinan con diferente peso en cada país, pero conforman un escenario particular del cambio en el comportamiento reproductivo latinoamericano.

En nuestra región, la influencia del Estado ha sido importante a la hora de modificar el tamaño deseado de la descendencia y sobre todo a la hora de aumentar la oferta de métodos anticonceptivos baratos, modificando así su demanda. El momento actual, sin embargo, necesita de un Estado que reoriente sus actividades y asuma los nuevos marcos conceptuales de política; para eso, los marcos institucionales de la mayoría de los países de la región pueden ser aprovechados y reformulados al mismo tiempo.

Los estudios enfocados en el caso de Brasil, por ejemplo, muestran como el Estado, a través de una serie de medidas que coexistieron con la ausencia de una política centralizada de planificación familiar, tuvo una influencia decisiva en el descenso de la TGF, de forma similar a lo sucedido en otros países de América Latina. Cabe insistir en que el descenso de la fecundidad en este contexto convivía y convive con la desigualdad propia del comportamiento reproductivo dual que ya fue mencionado y con riesgos y vulnerabilidades socioeconómicos y de salud (PERPÉTUO; RODRIGUEZ WONG, 2006). 
Ante todas estas peculiaridades, es necesario evitar la tentación de trasladar mecánicamente las experiencias europeas y resulta central atender los posibles efectos no deseados de las políticas, que pueden surgir si no se tienen en cuenta las peculiaridades de nuestro contexto. Los más evidentes son a) el riesgo de financiar la retirada de las mujeres del mercado laboral y b) la construcción de cuidados "para pobres” donde no exista la calidad y/o heterogeneidad suficiente como para promover el desarrollo inclusivo de los niños criados en contextos de exclusión. Pero también existe el riesgo de perpetuación de la llamada "doble jornada de trabajo" o doble carga femenina, en caso de que la conciliación suponga una mera "ayuda" para que las mujeres sigan a cargo de tareas productivas y reproductivas a la vez, sin verdadera corresponsabilidad de los hombres (y de instancias colectivas, estatales o comunitarias) en la crianza de los menores dependientes.

Los problemas de aplicabilidad de las medidas derivan además de la capacidad fiscal de los países. En ese terreno es difícil hacer recomendaciones firmes, ya que la carga de la financiación depende de opciones políticas más amplias. En cualquier caso, mencionadas las diferencias existentes, la racionalidad de las decisiones que toman los individuos, en el equilibrio entre uso del tiempo para la crianza y para el empleo, no difiere diametralmente de lo observado en otras regiones. Por ejemplo en relación a la incertidumbre económica: el desempleo aumenta la probabilidad de no tener nuevos hijos y la de posponerlos (ADSERA; MENENDEZ, 2009). Esa relación podría darse en América Latina en el entorno de una TGF estabilizada por debajo del reemplazo, como comienza a observarse en varios países (HERRERA LEON; CATASÚS, 2010). Lo que conocemos acerca del efecto de políticas universales o focalizadas en otros contextos, al menos en aquellos que comparten algunos aspectos de la "polarización reproductiva” (RENDALL et al., 2009) de nuestras poblaciones, puede ser asimismo de gran valor. Considerando todo lo dicho, veamos las principales decisiones de política familiar, sobre las que académicos y políticos pueden trabajar en conjunto.

\section{Las decisiones de política familiar}

Existen algunos puntos clave que vale la pena identificar, de modo de orientar el debate sobre el que deben actuar los tomadores de decisiones. Habrá quienes afirman que 1) no corresponde a los científicos la decisión política que deriva en un mandato posterior ("hay que hacer x"), pero sí 2) la alerta acerca del vínculo causal ("si se hace x, sucederá y"). Estamos de acuerdo en cuanto al primer punto. En relación al segundo, no sólo es necesario alertar acerca de estas conexiones causales, sino que también es necesario y legítimo pronunciarse acerca de cuáles son las decisiones centrales que hay que tomar, cuáles de ellas se enmarcan en la perspectiva de derechos, cuáles son los contextos en que "han funcionado" ciertas medidas en otros países, o qué vínculos pueden establecerse entre distintas dimensiones de política. Los puntos sobre los cuales es necesario expedirse, en nuestra opinión, son los siguientes: 
- es posible que deba optarse explícitamente por desfamiliarizar la crianza: es decir, dejar de apoyar la carga de la crianza en los recursos privados de las familias. Para eso, se deben suavizar las contradicciones entre maternidad/paternidad y desarrollo integral de la persona, promoviendo la incorporación de los varones a la tarea de la crianza de sus hijos. Cada medida, a su vez, puede ser escrutada desde criterios generales que evalúen su diseño (SOBOTKA, 2010);

- debe ser claro el objetivo principal de una política de fecundidad / familiar. Desde nuestro punto de vista, este objetivo no debiera ser el pronatalismo. No es prudente pensar instrumentos de política que apunten a un mero aumento de la Tasa Global de Fecundidad. Los motivos son básicamente dos: por un lado, a) no hay evidencia clara en la acumulación demográfica y demoeconómica que vincule el crecimiento poblacional per se con un mayor desarrollo; por otro, b) los intentos que se han basado en un marco decididamente pronatalista no han tenido mayor éxito en ese objetivo. La experiencia internacional muestra que la fecundidad ha aumentado en aquellos países que han implementado políticas que tenían otros objetivos;

- entonces, no creemos que la mirada más pertinente y eficiente sobre el tema sea aquella que apunta a "convencer" a las mujeres y varones a tener más hijos. Al contrario, consideramos que, desde la perspectiva de Derechos Humanos, el objetivo válido para instrumentar políticas familiares es colaborar para que mujeres y varones puedan construir su familia con el número de hijos que deseen, generando las condiciones para que los niños puedan desarrollar su crianza en las mejores circunstancias;

- en ese sentido, las políticas debieran tomar la experiencia europea y apuntar al marco que parece ser el de más alta probabilidad de éxito: la creación de condiciones duraderas que resulten favorables a la crianza de los niños, desde su nacimiento. Se trata de la generación de un "clima” institucional de protección a los hijos, de manera que su crianza sea corresponsabilidad de ambos padres trabajadores y otros actores sociales. Fundamentalmente, el Estado, aunque es recomendable incentivar la participación de otros actores sociales, que puedan colaborar y gestionar en cierta forma las medidas. Considerando que la mayoría de las intervenciones traerán consigo algún costo, cabe observar cuáles han mostrado mayor eficiencia allí donde han sido implementadas y así recomendar ciertas medidas sobre otras. Concretamente: las medidas de apoyo financiero, tales como el "cheque bebé" español, no parecen ser del todo significativas en términos de política familiary de fecundidad (tampoco en términos redistributivos si son de aplicación universal e independiente de los ingresos de cada hogar);

- por otra parte, es necesario insistir sobre la integralidad de los instrumentos de política. Políticas como las de empleo, género o vivienda otorgarán incentivos que pueden o no ir en la misma dirección que los de las políticas de fecundidad que se implementen, por lo que es necesario insertar cualquier innovación en un diseño coherente de medidas. Uno de los riesgos, de lo contrario, es la implementación de medidas inocuas o contraproducentes, con el consiguiente desaprovechamiento de recursos;

- debe favorecerse la provisión de cuidados infantiles, no solo mejorando su cobertura en términos de porcentaje de niños atendidos y su calidad, sino también diseñando sus horarios de manera a cumplir con objetivos de conciliación laboral - familiar para los padres. Para esto, los horarios pre y post educación formal deben estar cubiertos. Como puede verse, implementar esta mejora no implica necesariamente la creación de nuevas instituciones para los cuidados. A corto plazo, parece más razonable la apuesta por los cuidados externos al hogar (bajo la forma de centros de educación para la primera infancia) que por la implementación de cuidados internos (como existe para el caso de otros países, como Francia), con los costos que traería consigo, por ejemplo en formación de recursos humanos e institucionalización de la práctica. 


\section{Palabras finales}

De forma similar a lo que sucede con otras ciencias, la demografía está enfrentando un doble desafío: refinar sus herramientas de análisis e intervenir en el debate público, desde donde se le reclaman diagnósticos, prospectivas y miradas científico - políticas que permitan intervenir sobre las tendencias poblacionales.

Varios países de América Latina enfrentan un escenario de disminución de la fecundidad por debajo del nivel de reemplazo, ante el cual los gobiernos manifiestan su voluntad de intervención. En los próximos años, es altamente probable que todos los Estados que aún no lo hayan hecho incluyan en sus políticas de corto, mediano y largo plazo la mirada poblacional, así como políticas y programas específicos que pretendan influir sobre la fecundidad. ¿Qué posición debe adoptarse ante estas demandas, que vinculan la generación y difusión del trabajo académico con el uso de este trabajo con fines políticos (principalmente en el sentido de policy, aunque también de politics)?

En principio, la clarificación de los marcos conceptuales sobre los que se apoyan las medidas y la contextualización de "lo que se hace en otros países" es una tarea en la que cabe profundizar; se trata de comprender cuáles son los modelos de política disponibles, cuál es el perfil demográfico de los países en que tiene sentido su aplicación, cuáles son las derivaciones y posibles efectos no deseados, qué efectos inesperados puede acarrear, qué efectividad esperar de cada medida implementada y qué derivaciones tiene en relación con otras dimensiones de la vida social.

No sólo cabe advertir acerca de la complejidad de evaluar políticas y de la dificultad de implementar programas y medidas eficaces, sino que es necesario dar un paso más: tomar la experiencia de aquellos países europeos en los que un sistema estable y continuado de cuidados infantiles y medidas tendientes a lograr la modificación en los patrones de uso del tiempo ha hecho posible la creación de mejores condiciones de crianza para los niños y de satisfacción con la fecundidad para los padres y madres. En el mismo camino, tales países (acaso con Suecia como ejemplo principal) han logrado objetivos de equidad de género y eficiencia económica. Es necesario considerar esta experiencia para dar forma a las políticas necesarias en América Latina, adaptadas a las peculiaridades de nuestras poblaciones.

Así, las nuevas políticas familiares pueden lograr el objetivo de evitar una fecundidad de niveles "lowest-low" aun cuando los objetivos que se propongan explícitamente no sean los vinculados a la intensidad de la fecundidad sino a una variedad de otras metas, entre las cuales debe destacarse la igualdad de condiciones de crianza para los niños, incumplida en el habitual contexto de desigualdad social de nuestros países. Esto pone en primer plano el vínculo entre fecundidad y dimensiones de política tales como la de género, la de vivienda y la esfera de cuidados a miembros dependientes de los hogares.

\section{Referencias}

ADSERA, A. Where are the babies? Labor market conditions and fertility in Europe. European Journal of Population, v. 21, n. 1, p. 1-32, 2011.

ADSERA, A.; MENENDEZ, A. Fertility changes in Latin America in the context of economic uncertainty. IZA, 2009 (Discussion paper, n. 4019). 
BEGALL, K.; MILLS, M. The impact of subjective work control, job strain and work-family conflict on fertility intentions: a European comparison. European Journal of Population, v. 27, n. 4, p. 433-456, 2011.

BERNARDI, F. Public policies and low fertility: rationales for public intervention and a diagnosis for the Spanish case. Journal of European Social Policy, v. 15, n. 2, p. 123-138, 2005.

BJÖRKLUND, A. Does family policy affect fertility? Lessons from Sweden. Journal of Population Economics, v. 19, n. 1, p. 3-24, 2006.

BORRÀS, V.; TORNS, T.; MORENO, S. Las políticas de conciliación: políticas laborales versus políticas de tiempo. Papers, v. 83, p. 83-96, 2007.

CASTERLINE, J.; MENDOZA, J. Unwanted fertility in Latin America: historical trends, recent patterns. En: CAVENAGHI, S. (Ed.). Demographic transformations and inequalities in Latin America: historical trends and recent patterns. Rio de Janeiro: Alap, 2009, p. 193-218 (Serie Investigaciones, 8).

CASTRO MARTÍN, T.; CORTINA, C.; MARTÍN GARCÍA, T.; PARDO, I. Maternidad sin matrimonio en América Latina: análisis comparativo a partir de datos censales. Notas de Población, v. 93, p. 37-76, 2011.

CONNELLY, M. Fatal misconception. The struggle to control world population. Cambridge: Harvard University Press, 2008.

EHRLICH, P. The population bomb. New York: Ballantine, 1968.

ESPING-ANDERSEN, G. La política familiar y la nueva demografía. Información Comercial Española, V. 815, p. 45-60, 2004.

ESTEVE, A.; LESTHAEGHE, R.; LOPEZ-GAY, T. The Latin American cohabitation boom, 1970-2007. Population and Development Review, v. 38, n. 1, p. 55-82, 2012.

FLAQUER, L. Las políticas familiares en una perspectiva comparada. Barcelona: Fundación La Caixa, 2000 (Colección Estudios Sociales, n. 3).

FREJKA, T.; SARDON, J.-P. Childbearing trends and prospects in low-fertility countries: a cohort analysis. Dorbrecht: Kluwer Academic Publishers, 2004.

First birth trends in developed countries: persisting parenthood postponement. Demographic Research, v. 15, n. 6, p. 147-180, 2006.

GAUTHIER, A. H. The impact of family policies on fertility in industrialized countries: a review of the literature. Population Research and Policy Review, v. 26, n. 3, p. 323-346, 2007.

The State and the family: a comparative analysis of family. Policies in industrialized countries. Oxford: Clarendon Press, 1996.

HERRERA LEÓN, L.; CATASÚS, S. La fecundidad en Cuba entre 1970 y 2008: una reflexión a partir de escenarios y coyunturas socioeconómicas. Población y Salud en Mesoamérica, v. 8, n. 1, 2010.

HOEM, J. Why does Sweden have such high fertility? Demographic Research, v. 13, n. 22, p. 559-572, 2005.

KALWIJ, A. The impact of family policy expenditures on fertility in Western Europe. Demography, v. 47, n. 2, p. 503-519, 2010.

KIPPEN, R. Trends in age and parity specific fertility in Australia. ANU Demography and Sociology Program, 2003 (Working papers in demography, n. 91).

LESTHAEGHE, R.; VAN DE KAA, D. J. Twee demografische transities? (two demographic transitions?). En: VAN DE KAA, D. J.;. LESTHAEGHE, R. (Eds.). Bevolking: groei en krimp (population: growth and decline). Deventer: Van Loghum Slaterus, 1986, p. 9-24.

LUTZ W.; SKIRBEKK, V.;TESTA, M. R. The low fertility trap hypothesis: forces that may lead to further postponement and fewer births in Europe. IIASA Interim Report IR-06-01, 2006. 
MCDONALD, P. An assessment of policies that support having children from the perspectives of equity, efficiency and efficacy; Vienna yearbook of population research. 2006, p. 213-234.

MCDONALD, P.; KIPPEN, R. Measuring the quantum of fertility during a long-term shift from early to late childbearing: Australia 1946-2007. In: INTERNATIONAL POPULATION CONFERENCE. Marrakech, 2009.

OLÁH, L. S.; BERNHARDT, E. M. Sweden: combining childbearing and gender equality. Demographic Research, v. 19, n. 28, p. 1105-1144, 2008.

PERI, A.; PARDO, I. Nueva evidencia sobre la hipótesis de la doble insatisfacción en Uruguay: ¿cuán lejos estamos de que toda la fecundidad sea deseada? Montevideo: UNFPA, 2008.

PERPÉTUO, I.; RODRÍGUEZ WONG, L. Hacia una tasa de reemplazo: programas y políticas que afectaron el curso de la fecundidad en Brasil. Papeles de Población, v. 47, p. 243-275, 2006.

REHER, D. Towards long-term population decline: a discussion of relevant issues. European Journal of Population, v. 23, p. 189-207, 2007.

RENDALL, M.; EKERT-JAFFÉ, O.; JOSHI, H.; LYNCH, K.; MOUGIN, R. Universal versus economically polarized change in age at first birth: a French-British comparison. Population and Development Review, v. 35, n. 1, p. 89-115, 2009.

ROBINSON, W.; ROSS, J. The global family planning revolution: three decades of population policies and programs. Washington DC: World Bank Publications, 2007.

ROSERO-BIXBY, L. Comment: Population Programs and Fertility. Population and Development Review, v. 27, Supplement: Global Fertility Transition, p. 205-209, 2001.

ROSERO-BIXBY, L.; CASTRO-MARTÍN, T.; MARTÍN GARCÍA, T. Is Latin America starting to retreat from early and universal childbearing? Demographic Research, v. 20, n. 9, p. 169-194, 2009.

ROSSY, K. Politicizing pronatalism: exploring the Nazi ideology of women through the lens of visual propaganda, 1933-1939. The Graduate History Review, v. 3, n. 1, p. 49-77, 2011.

SHREFFLER, K. M.; PIRRETTI, A. E.; DRAGO, R. Work-family conflict and fertility intentions: does gender matter? Journal of Family and Economic Issues, v. 31, p. 228-240, 2010.

SOBOTKA, T. Low fertility in Europe and beyond: trends, consequences and government actions. In: UNFPA TECHNICAL MEETING ON LOW FERTILITY, AGEING AND POPULATION DECLINE. Viena. 2010.

Groningen, 2004.

Posponement of childbearing and low fertility in Europe. Amsterdam: Rijks Universiteit

Does fertility respond to work and family-life reconciliation policies in France? In: TAKAYAMA, N.; WERDING , M. (Eds.). Fertility and public policy: how to reverse the trend of declining birth rates. Cambridge, MA and London, UK: MIT-Press, 2009.

TAKAYAMA, N.; WERDING, M. (Eds.). Fertility and public policy: how to reverse the trend of declining birth rates. Cambridge MA and London UK: MIT-Press, 2009.

THÉVENON, O. Family policies in OECD countries: a comparative analysis. Population and Development Review, v. 37, n. 1, p. 57-87, 2011.

THÉVENON, O. Does fertility respond to work and family-life reconciliation policies in France? In: TAKAYAMA, N.; WERDING, M. (Eds.). Fertility and public policy: how to reverse the trend of declining birth rates. Cambridge MA and London UK: MIT-Press, capítulo 10, 2009.

UNDP. América Latina: gestión de conocimiento para la igualdad de género. Disponible en: en<http://www. americalatinagenera.org/es/index.php?option=com_content\&view=ar ticlE\&ID=231\&ITEMID=160 . Acceso el: 10 mar. 2012.

VARELA, C.; PARDO, I.; POLLERO, R. Políticas de fecundidad: sistematización de experiencias y recomendaciones para el caso uruguayo. Montevideo: CSP - OPP, 2011. 


\title{
Autores
}

Ignacio Pardo es doctor en Sociología (Universidad Complutense de Madrid) y docente - investigador en el Programa de Población, Facultad de Ciencias Sociales - UdelaR (Uruguay).

Carmen Varela es socióloga, con especialización en demografía (CELADE). Profesora Adjunta e investigadora, Coordinadora del Programa de Población, Facultad de Ciencias Sociales - UdelaR (Uruguay).

\section{Resumo}

Fecundidade, taxa de reposição e políticas familiares na América Latina e no Caribe: o que se pode apreender da experiência europeia?

A queda da fecundidade nos países da América Latina e do Caribe é um fenômeno inédito e veloz. Numerosos países estão abaixo do nível da taxa de reposição, incluindo casos emblemáticos como o do Brasil. É necessário compreender a partir de que marco conceitual podem-se harmonizar as diferentes medidas de política pública que estão surgindo e irão surgir no futuro, tendo como referência as questões de família e fecundidade baixa. Além disso, cabe resumir as experiências daqueles países nos quais se implantaram políticas familiares em contexto de fecundidade abaixo da reposição. 0 artigo resume e analisa as políticas aplicadas na Suécia, Espanha e França e discute em que medida estas podem ser úteis para a formulação de políticas na América Latina. Também sugere que um conjunto de decisões-chave deve ser tomado no momento de se desenharem políticas familiares em tal contexto novo para nossa região.

Palavras-chave: Fecundidade. Políticas familiares. Fecundidade abaixo da reposição. América Latina e Caribe.

\begin{abstract}
Below-replacement fertility and family policy in Latin America and the Caribbean: learning from the European experience

In recent years fertility in Latin America and the Caribbean has fallen at a very rapid pace. In many countries it fell to below-replacement levels, including emblematic cases such as Brazil. In this context, it is important to analyze the conceptual backgrounds that can give coherence to different policy measures. It is also essential to understand and analyze family policies in other countries having below-replacement fertility levels. In this paper we consider the cases of Sweden, Spain and France and discuss to what extent those policies can serve as inspirations for policy makers in Latin America and the Caribbean. We also suggest a list of key decisions needed to carry forward the design of family policy measures in this low-fertility context, which is unprecedented in our region.
\end{abstract}

Keywords: Fertility. Family policy. Below-replacement fertility. Latin America and the Caribbean. 